$17^{\text {th }}$ International Congress of Metrology, 02005 (2015)

DOI: $10.1051 /$ metrology $/ 201502005$

(C) Owned by the authors, published by EDP Sciences, 2015

\title{
Possibility and probability: application examples and comparison of two different approaches to uncertainty evaluation
}

\author{
Alessandro Ferrero ${ }^{1,1}$, Jean-Michel $\mathrm{Pou}^{2}$, Marco Prioli ${ }^{1}$, and Simona Salicone ${ }^{1}$ \\ ${ }^{1}$ PolitecnicodiMilano,DipartimentodiElettronica,InformazioneeBioingegneria,P.zaLeonardodaVinci32,20133Milano,Italy \\ ${ }^{2}$ DeltaMu,63800-Cournond'Auvergne,France
}

\begin{abstract}
This paper proposes two interesting applications of the approach to uncertainty evaluation and representation in terms of Random-Fuzzy Variables. One covers the expression of the calibration uncertainty of gauge blocks, and one considers unknown temperature variations, with respect to temperature at calibration time, in expressing a voltmeter uncertainty. Both considered examples show that the proposed approach is more effective than the traditional GUM approach.
\end{abstract}

\section{Introduction}

The evaluation and expression of measurement uncertainty is still an hot and debated topic, despite the uncertainty concept, as defined by the Guide to the Expression of Uncertainty in Measurement (GUM) [1, 2], has been universally accepted by the metrologists. The focus of the discussion is on the validity of the mathematical framework, probability, into which the evaluation of measurement uncertainty has been confined.

There are several cases, in the everyday measurement practice, in which the validity of a purely probabilistic approach can be doubted, for instance every time a systematic effect has to be considered, whose value is unknown, but the interval into which it is supposed to lie. Let's consider the acceleration of gravity, $\mathrm{g}$, in a given location. Due to unknown measurement errors, we cannot know its true value, though we can assume an interval into which the local $g$ is supposed to reasonably lie. On the other hand, when using the measured g value, we cannot consider it as a random variable in the estimated interval, because $\mathrm{g}$, in the considered location, does not vary, and, hence, it represents an unknown and uncompensated systematic effect.

In order to represent such effects and their combination with other random and non-random effects in a more correct and effective way, a new mathematical framework has been proposed in the recent years [3-6], based on the theories of evidence and possibility, that generalize probability and allow also non-random effects to be handled. While the theoretical framework has been well developed [6], few practical applications have been considered, up to now. This paper, after having briefly recalled the very fundamentals of uncertainty expression in terms of possibility distributions, is aimed at showing the effectiveness of this new approach in two simple, though significant cases, where unknown and uncompensated systematic effects may cause the GUM approach to provide incorrect results. The first one considers the expression of calibration uncertainty for gauge blocks, whilst the second one considers unknown temperature variations, with respect to temperature at calibration time, in expressing a voltmeter uncertainty.

\section{Overview of the RFV approach}

The employed mathematical framework, based on the possibility theory, considers possibility distributions, instead of probability distributions, to represent the distribution of values that can be reasonably attributed to the measurand. Without entering too many mathematical details, for which the reader is addressed to [6, 7], a possibility distribution (PD) is defined as a function $r$ over a support $X$

$$
r: X \rightarrow[0,1]
$$

such that:

$$
\sup (r(x))=1
$$

\footnotetext{
${ }^{1}$ Corresponding author: alessandro.ferrero@polimi.it
} 
The cuts $I_{\alpha}$ of a PD, called $\alpha$-cuts and defined as:

$$
I_{\alpha}=\{x \mid r(x) \geq \alpha\},
$$

can be considered as a generalization of the probabilistic concept of confidence intervals and a credibility that an element $x$ belongs to them can be associated to each interval, in the range $[0,1][6,8]$. In particular, the credibility value is given, for each $\alpha$-cut at level $\alpha$, by 1 $\alpha$ [7]. Therefore, expressing a measurement result in terms of a PD yields a family of confidence intervals at various confidence levels. Since the final aim of uncertainty evaluation is "to provide

an interval about the result of a measurement within which the values that could reasonably be attributed to the measurand may be expected to lie with a high level of confidence" [1], expressing a measurement result in terms of a

$\mathrm{PD}$ is in complete agreement with the requirements of the GUM.

It has been proved [6] that a PD can effectively represent the effect of any kind of contributions to uncertainty. However, different effects (random and nonrandom) give different contributions to uncertainty and combine in different ways. Therefore, we may expect that a single PD is not enough to represent all effects and their combinations, and that an aggregation of at least two PDs is required.

Random Fuzzy Variables (RFVs) have been defined to this purpose [7]. An RFV is defined by two possibility distributions: the internal one $r_{\text {int }}(x)$ considers all nonrandom contributions to uncertainty, whilst the external one $r_{\text {ext }}(x)$ considers also the random contributions. The external PD is obtained by combining the internal PD $r_{\text {int }}(x)$ with the random PD $r_{\text {ran }}(x)$, which considers only the random contributions to uncertainty [7].

Fig. 1 shows an example of RFV and its PDs.

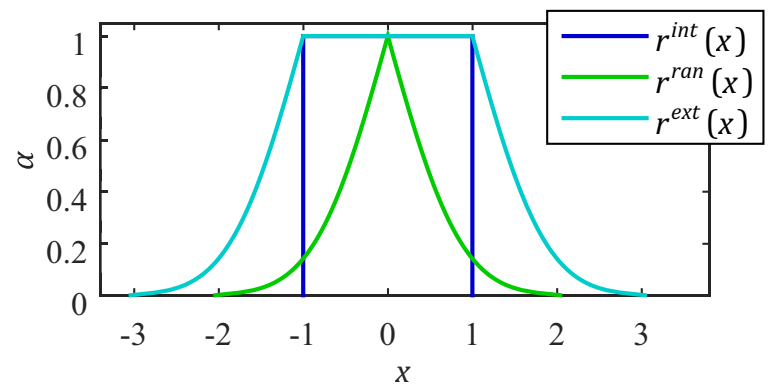

Figure 1. PDs composing an RFV (blue and cyan lines).

Since the two PDs $r_{\text {int }}(x)$ and $r_{\text {ran }}(x)$ represent different effects that combine in different ways, the mathematics used to combine RFVs must consider the different characteristics of these two PDs. This mathematics exploits the definition of joint PDs, given in [9] for the internal PDs and in [10] for the random PDs, and the application of the Zadeh's extension principle [11], as also shown in $[12,13]$, to which the reader is addressed for further details.

\section{Uncertainty evaluation for gauge blocks}

As a first example, let us consider one of the examples proposed by the Collège Français de Métrologie (CFM), and in particular the $27^{\text {th }}$ example on the calibration of gauge blocks [14]. In this example, different uncertainty sources are considered, as listed in Table 1.

According to the CFM example, all effects are considered as random ones, and a specific probability density function (PDF) is assumed for each of them, together with their standard deviation, as listed in Table 1. Following the approach of the GUM [1], being those effects not correlated, a combined standard uncertainty $u_{\mathrm{c}}$ $=32 \mathrm{~nm}$ is obtained [14], thus leading to a combined expanded uncertainty $U_{c}=64 \mathrm{~nm}$, under the assumption of a normal

\begin{tabular}{|c|l|c|c|}
\hline$\#$ & Source & PDF & $u(\cdot)$ \\
\hline 1 & repeatability & $\mathrm{N}$ & $16 \mathrm{~nm}$ \\
\hline 2 & accuracy correction & $\mathrm{N}$ & $4 \mathrm{~nm}$ \\
\hline 3 & quantization error & $\mathrm{T}$ & $4.08 \mathrm{~nm}$ \\
\hline 4 & comparator geometry & $\mathrm{U}$ & $5.77 \mathrm{~nm}$ \\
\hline 5 & length of the reference block & $\mathrm{N}$ & $11 \mathrm{~nm}$ \\
\hline 6 & reference drift between calibrations & $\mathrm{N}$ & $6.66 \mathrm{~nm}$ \\
\hline 7 & temperature deviation between blocks & $\mathrm{U}$ & $17 \mathrm{~nm}$ \\
\hline 8 & $\begin{array}{l}\text { difference in the temperature } \\
\text { coefficients }\end{array}$ & $\mathrm{N}$ & $7 \mathrm{~nm}$ \\
\hline 9 & contact deformation on the reference & $\mathrm{N}$ & $10 \mathrm{~nm}$ \\
\hline 10 & contact deformation on the DUC & $\mathrm{N}$ & $10 \mathrm{~nm}$ \\
\hline
\end{tabular}

Table 1. Contributions to calibration uncertainty. N: normal PDF. T: triangular PDF. U: uniform PDF.

PDF of the final result and a coverage factor $k=2$, corresponding to an interval with $95 \%$ confidence level.

However, some of these effects, namely those in rows 2, 4, 5, 9 and 10 in Table 1, show a systematic behavior, since they originate in the imperfections of the measurement process at calibration time. As a matter of fact, the length of the gauge blocks, their geometry, the deformation on the contact point and the accuracy of the sensors, at calibration time, are not random quantities. We simply cannot know their exact values, that are assumed to take single values inside given intervals. When the calibrated block gauge is used in a measurement process, this lack of knowledge cannot be considered as a random effect affecting the measurement result, since the length of the gauge blocks, their geometry, the deformation on the contact point and the accuracy of the sensors do not vary during the measurement procedure. Therefore, this lack of knowledge represents a systematic effect. Hence, just adding their assumed variances to obtain the combined uncertainty, as stated by the GUM, makes little sense.

The representation of the calibration result in terms of an RFV, briefly recalled in the previous section, appears to be much more effective, since it allows one to consider 
the systematic effects in the internal PD, separated from the random ones. Therefore, starting from the available information listed in Table 1, considering the contribution coming from row 4 as total ignorance on the related error, and according to the procedure shown in [15], the RFV shown in Fig. 2 is obtained, where the contributions in rows 2, 4, 5, 9 and 10 have been used to build the internal $\mathrm{PD}$, and the other ones have been used to build the random PD.

It can be noted that the $\alpha$-cut at $\alpha=0.32$, corresponding to the interval with $68 \%$ confidence level, has an halfwidth of $0.06 \mu \mathrm{m}$ and the $\alpha$-cut at $\alpha=0.05$, corresponding to the interval with $95 \%$ confidence level, has an halfwidth of $0.11 \mu \mathrm{m}$. In GUM terms, this value corresponds to the combined expanded uncertainty, associated to the confidence interval with $95 \%$ confidence level. Therefore, it is possible to compare this value with the combined expanded uncertainty $U_{c}$ evaluated by the CFM. It follows that the RFV approach provides a larger value, as

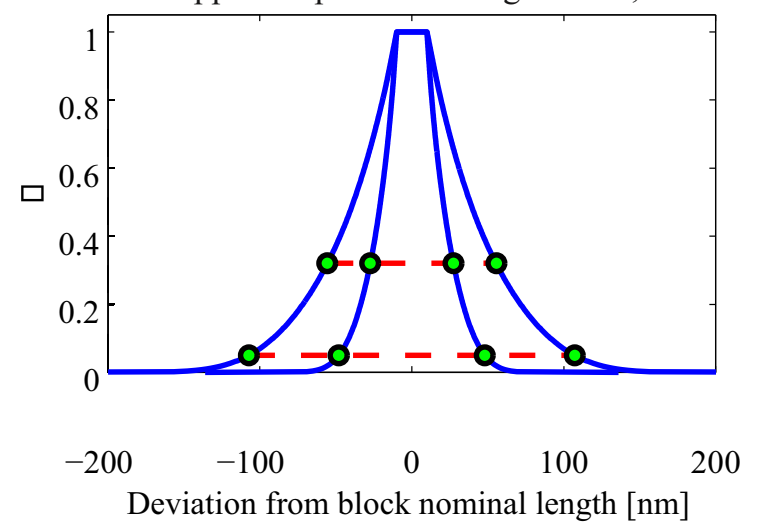

Figure 2. RFV representing the calibration result of a $50 \mathrm{~mm}$ gauge block in terms of deviation from the nominal value. The two intervals are those at $68 \%$ (upper interval) and $95 \%$ (lower interval) confidence level.

expected, since it takes into account the systematic behavior of some considered effects.

\section{Uncertainty evaluation for a voltmeter}

This second measurement example consists in the calibration of a Fluke 8845A digital multimeter (device under calibration, DUC), in the DC voltage measurement, by comparison with a Fluke $8508 \mathrm{~A}$ reference multimeter (standard). To do that, a relationship has to be established between the values measured by the standard and the corresponding indications of the DUC.

Moreover, considering the uncertainty contributions affecting the standard and the calibration process, an accuracy estimate needs to be associated with the DUC. This can be done by following three different approaches: the GUM approach reported in [1], the Monte Carlo (MC) simulations, as suggested in [2], or the RFV approach. The main goal of this example is to show that, following the

${ }^{1}$ For the sake of simplicity, the calibration procedure is here performed for a single voltage value. Of course, the procedure may be
RFV approach, a more informative accuracy estimate is obtained. Moreover, the RFV approach allows a more effective accuracy estimate than the GUM and MC approaches when the voltmeter is operated, after calibration, under unknown temperature conditions, that are likely to be different than the temperature at calibration time.

The employed calibration procedure is the following: the same voltage ${ }^{1}$ is simultaneously measured by the standard, with a fixed operating temperature $\vartheta_{S}=23^{\circ} \mathrm{C}$, and the DUC, for different operating temperatures in the range $8^{\circ} \mathrm{C} \leq \vartheta \leq 38^{\circ} \mathrm{C}$. The obtained expected value of the voltage is, according to the standard, ${ }^{-} v_{S}=9.999942 \mathrm{~V}$, while multiple expected values ${ }^{-} v_{D U C}(\vartheta)$ are obtained according to the indications of the DUC, as shown in Table 2.

Starting from ${ }^{-} v_{S}$ and ${ }^{-} v_{D U C}(\vartheta)$, a correction value $\delta^{-}$ (७) can be obtained, as:

$$
\delta^{-}(\vartheta)=\mathrm{v}^{-}-\mathrm{v}^{-} D U C(\vartheta)
$$

Table 2. $v^{-}{ }_{D U C(\vartheta)}$ and $\sigma_{\mathrm{v} D U C(\vartheta)}$ for different $\vartheta$ values.

\begin{tabular}{ccc}
$\vartheta\left[{ }^{\circ} \mathrm{C}\right]$ & $\mathrm{v}^{-}{ }_{D U C(\vartheta)}[\mathrm{V}]$ & $\sigma_{\mathrm{v} D U C}(\vartheta)[\mu \mathrm{V}]$ \\
\hline 8 & 9.99991 & 9 \\
18 & 9.99990 & 8 \\
& & \\
28 & 9.99989 & 8 \\
38 & 9.99988 & 7
\end{tabular}

The evaluation of (4) completes the calibration procedure. Then, when the calibrated multimeter is employed to measure a voltage $\mathrm{v}_{m}$, the correction $\delta^{-}(\vartheta)$ can be applied according to:

$$
\mathrm{v}=\mathrm{v}_{m}+\delta^{-}(\vartheta)
$$

Of course, due to the uncertainty affecting ${ }^{-} \mathrm{v}_{S}$ and $\mathrm{v}^{-}{ }_{D U C}(\vartheta)$, also the correction $\delta^{-}(\vartheta)$ is affected by uncertainty. Evaluating this last uncertainty contribution corresponds to estimate the residual uncertainty contributions associated with the calibrated voltmeter, i.e. its accuracy. In fact, according to (5), for a specific measured value $\mathrm{v}_{m}$ and a specific operating temperature $\vartheta$, the uncertainty affecting the corrected value $v$ is equal to the uncertainty affecting $\delta^{-}(\vartheta)$.

The uncertainty contributions involved in the calibration process are a type B contribution provided by the manufacturer of the standard $\left(\Delta_{v_{S}}=34 \mu \mathrm{V}\right)$, a type A contribution due to the experimental variability of $v_{S}\left(\sigma_{v_{S}}=\right.$ $4 \mu \mathrm{V})$ and a type A contribution due to the experimental variability of ${ }^{-} \mathrm{v}_{D U C}(\vartheta)$, as shown in Table 2 . Moreover, when the operating temperature of the calibrated voltmeter 
is considered unknown, a type B contribution is added to the final accuracy estimate of the voltmeter.

\subsection{GUM approach}

According to the GUM approach [1], the combined uncertainty $u_{\delta(\vartheta)}$ can be found by combining the standard uncertainties of $v_{S}$ and $v_{D U C}$ through (4), starting from some assumptions about their probability density functions (PDFs). In particular, the standard uncertainty of $v_{S}$ is obtained assuming a uniform PDF of width $2 \Delta_{v_{S}}$ and a normal PDF with standard deviation $\sigma_{v_{S}}$, while the standard uncertainty of $\mathrm{v}_{D U C}$ is obtained assuming a normal

PDF with standard deviation $\sigma_{\mathrm{vDUC}}(\vartheta)$. Then, according to (5), for a given measured value $v_{m}$ and a specific operating temperature $\vartheta$, the uncertainty $u_{\mathrm{v}}$ is equal to $u_{\delta(\vartheta)}$.

Another uncertainty contribution should be added in the evaluation of $u_{\mathrm{v}}$ in the case of an unknown operating temperature of the calibrated voltmeter in a given range. As an example, let us assume that during a measurement process after calibration the operating temperature may assume a constant, though unpredictable value in the range $18^{\circ} \mathrm{C} \leq \vartheta \leq 28^{\circ} \mathrm{C}$. This means that the best estimate of the correction $\delta^{-}(\vartheta)$ to be applied in (5) is now unknown. Following the GUM probabilistic approach, the only way to include this contribution to uncertainty is to consider an additional random contribution in the evaluation of $u_{\mathrm{v}}$. In particular, the additional contribution is obtained by considering a uniform PDF of width:

$$
2 \Delta_{\delta}=\delta^{-}\left(28^{\circ} \mathrm{C}\right)-\delta^{-}\left(18^{\circ} \mathrm{C}\right)
$$

The main problem with this approach is that, both for fixed and unknown operating temperatures, the resulting PDF of $v$ is unknown and, therefore, the confidence for which $\mathrm{v}$ is supposed to lie in the interval $\left[\mathrm{v}-K \cdot u_{\mathrm{v}}, \mathrm{v}+K\right.$. $\left.u_{\mathrm{v}}\right]$ is unknown, where $K$ is the coverage factor. This confidence may be determined by referring to the central limit theorem (CLT), even if few input variables have been combined. If an higher accuracy in the uncertainty evaluation is desired, Monte Carlo (MC) simulations should be considered, as suggested by GUM supplement 1 [2]. In this case, the samples of $v$ are directly obtained and they provide an estimate of the PDF of v. In Sec. 4.3, this PDF will be transformed into an equivalent PD [8] that will be compared with the ones obtained following the RFV approach.

\subsection{RFV approach}

The calibration procedure can be also modeled following the RFV approach. Considering the uncertainty contributions and associated PDFs discussed above, RFVs can be associated with $v_{S}$ and $v_{D U C}(\vartheta)$, as shown in Fig. 3 and 4 , respectively. Since only random contributions are considered for $\mathrm{v}_{D U C}(\vartheta)$, its RFVs are composed by the sole random PDs $r_{\mathrm{v} D U C} \mid \vartheta$.

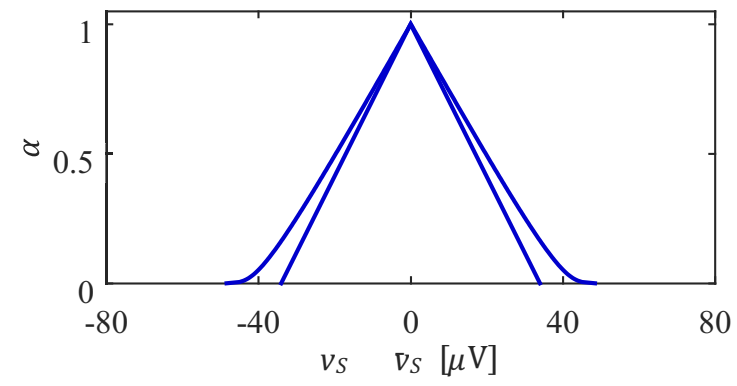

Figure 3. RFV associated with $v_{S}$.

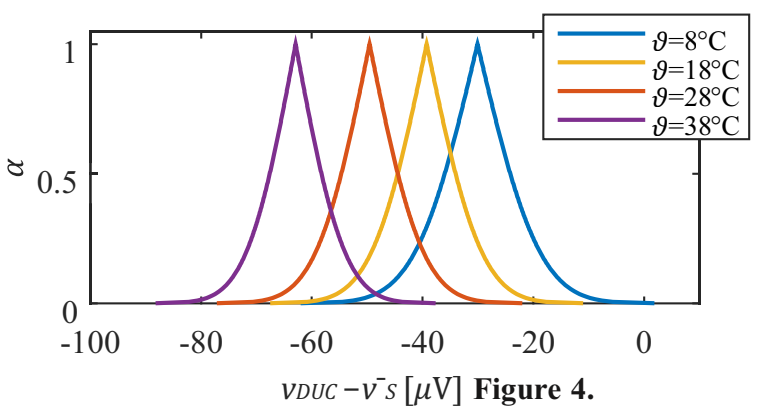

RFVs associated with $\mathrm{v}_{D U C}(\vartheta)$.

Due to the uncertainty presence, for each temperature $\vartheta$, an RFV shall be associated with $\delta(\vartheta)$ given by the difference between the RFVs associated with $v_{S}$ and

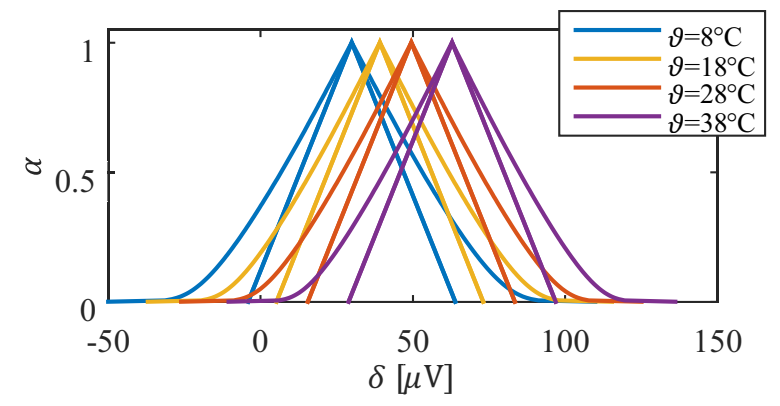

Figure 5. RFVs associated with $\delta(\vartheta)$.

$\mathrm{v}_{D U C}(\vartheta)$. The resulting internal and external PDs $r \delta \mid \vartheta$ composing these RFVs are shown in Fig. 5.

The PDs $r_{\delta \mid \vartheta}$ can be usefully employed to obtain, for a given measured voltage $v_{m}$ and temperature value $\vartheta$, the PDs of the corrected voltage values v. In fact, according to (5), the PDs $r_{\mathrm{v} \mid\left(v_{m}, \vartheta\right)}$ can be simply obtained, as:

$$
r \mathrm{v}|(\mathrm{v} m, \vartheta)=r \delta| \vartheta(\mathrm{v}-\mathrm{V} m(\vartheta))
$$

As an example, the RFV of $\mathrm{v}$ for specific values $\mathrm{v}_{m}=$ $9.99990 \mathrm{~V}$ and $\vartheta=23^{\circ} \mathrm{C}$ is shown in Fig. 6 (red lines). This figure proves that, after the correction, the most possible value of $v$ is, as expected, ${ }^{-} v_{S}$. 


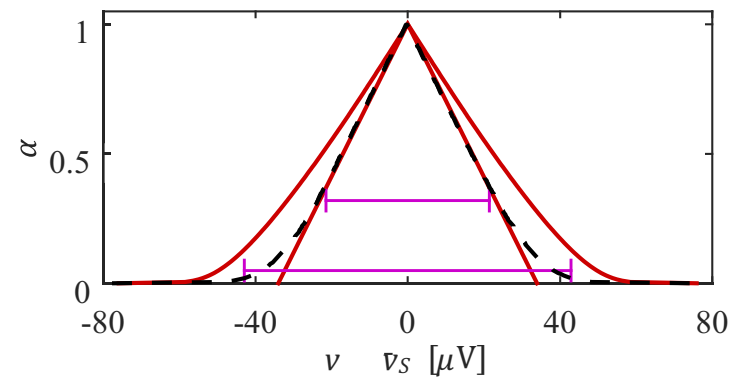

Figure 6. RFV (red lines), PD induced by MC simulations (black dashed lines), and confidence intervals provided by GUM approach and CLT (magenta lines) associated with $v$ for $\vartheta=23^{\circ} \mathrm{C}$.

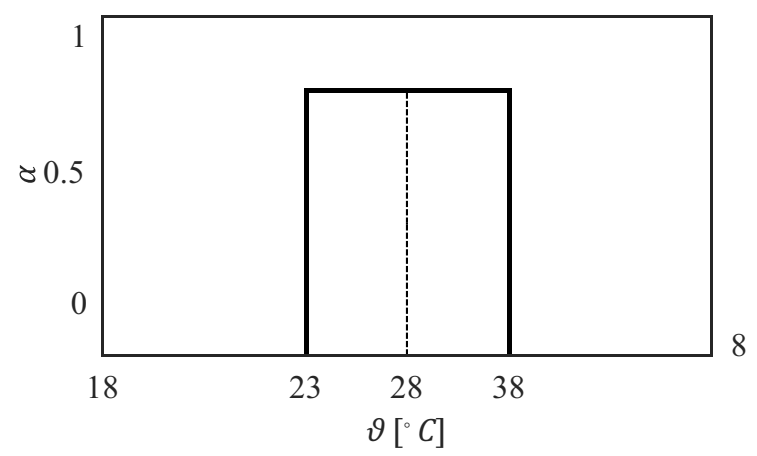

Figure 7. RFV associated with $\vartheta$

The availability of PDs $r_{\mathrm{v} \mid\left(\mathrm{v}_{m}, \vartheta\right)}$ allows one to include in the accuracy estimate the effect of an unknown operating temperature in a given range. A uniform PD expressing the total ignorance about temperature in the range $18^{\circ} \mathrm{C} \leq \vartheta \leq$ $28^{\circ} \mathrm{C}$ can be associated with $\vartheta$, as shown in Fig. 7.

According to [16], a principle can be followed to include the information provided by $r \vartheta$ in the accuracy estimate:

$$
r v|v m=\sup T \min r v|(v m, \vartheta), r \vartheta
$$

i.e. $r_{v} \mid v_{m}$ are the marginal PDs of the joint PDs between $r_{\mathrm{v} \mid\left(\mathrm{v}_{m}, \vartheta\right)}$ and $r_{\vartheta}[16]$. The resulting PDs $r_{\mathrm{v} \mid \mathrm{v}_{m}}$ are shown in Fig. 8 (green lines). Of course, an unknown operating temperature in a given range produces an increment of the non-random uncertainty contributions.

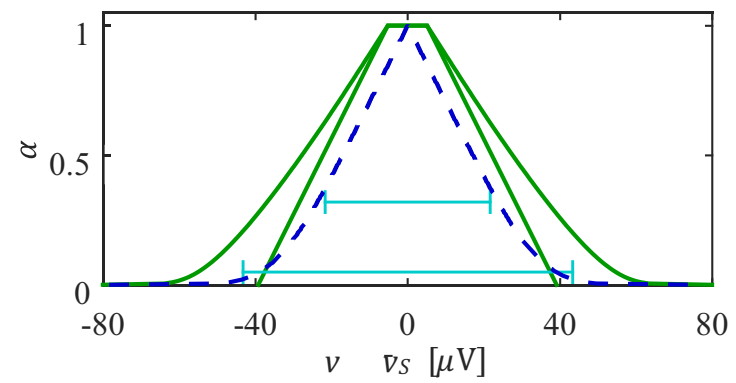

Figure 8. RFV (red lines), PD induced by MC simulations (black dashed lines), and confidence intervals provided by GUM approach and CLT (magenta lines) associated with $v$ for $18^{\circ} \mathrm{C} \leq$ $\vartheta \leq 28^{\circ} \mathrm{C}$.

\subsection{Comparison}

The results obtained about the possible corrected values $\mathrm{v}$ following the GUM approach and considering the CLT, following the MC approach, and applying the RFV approach, are compared in Figs. 6 and 8. In particular, for the MC simulations, equivalent PDs of $v$ are obtained (black and blue dashed lines), while for the GUM and CLT approach, the $68 \%$ and $95 \%$ confidence intervals are shown (magenta and cyan horizontal lines). To simplify the comparison, the $68 \%$ and $95 \%$ confidence intervals obtained following the thee approaches are reported in Tables 3 and 4. For the RFV approach, type- 2 confidence intervals are obtained.

Table 3. Confidence intervals of $v$ for $\vartheta=23^{\circ} \mathrm{C}$.

\begin{tabular}{c|cc} 
& $68 \%$ conf. int. $[\mu \mathrm{V}]$ & $95 \%$ conf. int. $[\mu \mathrm{V}]$ \\
\hline CLT & {$[-21,21]$} & {$[-43,43]$} \\
MC & {$[-24,24]$} & {$[-39,39]$} \\
RFV & {$[[-32,-23],[23,32]]$} & {$[[-50,-32],[32$,} \\
& & $50]]$
\end{tabular}

Table 4. Confidence intervals of $v$ for $18^{\circ} \mathrm{C} \leq \vartheta \leq 28^{\circ} \mathrm{C}$.

\begin{tabular}{c|cc} 
& $68 \%$ conf. int. $[\mu \mathrm{V}]$ & $95 \%$ conf. int. $[\mu \mathrm{V}]$ \\
\hline CLT & {$[-22,22]$} & {$[-44,44]$} \\
MC & {$[-24,24]$} & {$[-40,40]$} \\
RFV & {$[[-37,-28],[28,37]]$} & {$[[-55,-38],[38$,} \\
& & $55]]$
\end{tabular}

When a known temperature $\vartheta=23^{\circ} \mathrm{C}$ is considered (Fig. 6 and Table 3), the MC and RFV approaches provide compatible results. In fact, all the MC confidence intervals fall inside the RFV type- 2 confidence intervals. In this respect, the RFV approach leads to a more informative

result since it considers the different random and nonrandom nature of the contributions to uncertainty, thus providing an uncertainty estimate due to the sole nonrandom effects (narrowest confidence intervals) and an estimate due to all effects (largest confidence intervals). On the other hand, the GUM and CLT approach provides only approximate results.

When an unknown temperature in the range $18^{\circ} \mathrm{C} \leq \vartheta$ $\leq 28^{\circ} \mathrm{C}$ is considered (Fig. 8 and Table 4 ), the MC and RFV approaches provide different results. This is due to a different representation of the available knowledge about the unknown temperature. Following the GUM and CLT approach and the MC approach, the effect of an unknown temperature is modeled as an additive random contribution. However, the effect of an unknown temperature is not random, but systematic, since it produces a (unknown) deviation of the most possible value of the correction $\delta^{-}(\vartheta)$. Moreover, following these approaches, a specific PDF (uniform) is associated with temperature, even if the knowledge about the specific PDF is not available, as in the considered example. On the other hand, the systematic nature and the unknown PDF of temperature are correctly represented by following the RFV approach. According to Fig. 8 and Table 4, when the 
GUM and CLT approach and the MC approach are erroneously applied, an underestimate of uncertainty may be obtained.

\section{Conclusions}

The application of the RFV approach to uncertainty evaluation in two practical examples has been considered by this paper and compared with the results provided by the application of the GUM and its supplement 1.

The obtained results confirm that the RFV approach extends the purely probabilistic GUM approach and allows one to handle and combine also the contributions to uncertainty given by uncompensated systematic effects, when the only available knowledge related to these contributions is an interval into which they are supposed to lie.

Therefore, the considered examples confirm that the RFV approach is a promising way to evaluate uncertainty in several practical applications when the systematic effects are not completely known and, hence, cannot be compensated for.

\section{References}

[1] JCGM 100:2008, Evaluation of Measurement Data - Guide to the Expression of Uncertainty in Measurement, (GUM 1995 with minor corrections), Joint Committee for Guides in Metrology (2008)

[2] JCGM 101:2008, Evaluation of measurement data - Supplement 1 to the Guide to the expression of uncertainty in measurement - Propagation of distributions using a Monte Carlo method, Joint Committee for Guides in Metrology (2008)

[3] G. Mauris, V. Lasserre, L. Foulloy, Measurement 29, 165 (2001)

[4] M. Urbanski, J. Wasowsky, Measurement, Elsevier Science 34, 67 (2003)

[5] A. Ferrero, S. Salicone, The theory of evidence for the expression of uncertainty in measurement, in 12 International Metrology Congress (Lyon, France, June 20-23, 2005)

[6] A. Ferrero, S. Salicone, IEEE Trans. Instrum. Meas. 61, 2167 (2012)

[7] S. Salicone, Measurement Uncertainty: an approach via the mathematical theory of evidence, Springer series in reliability engineering (Springer, New York, NY, USA, 2007), ISBN 0387306552

[8] A. Ferrero, M. Prioli, S. Salicone, B. Vantaggi, IEEE Trans. Instrum. Meas. 62, 982 (2013)

[9] A. Ferrero, M. Prioli, S. Salicone, IEEE Trans. Instrum. Meas. 62, 720 (2013)

[10] A. Ferrero, M. Prioli, S. Salicone, IEEE Trans. Instrum. Meas. 63, 80 (2014)

[11] L.A. Zadeh, Fuzzy Sets and Systems 1, 3 (1978)

[12] A.Ferrero, M. Prioli, S. Salicone, Uncertainty evaluation by means of joint possibility distributions, in 16 International Congress of Metrology (Paris, France, 2013)

[13] A.Ferrero, M. Prioli, S. Salicone, A measurement application of conditional possibility distributions, in I2MTC 2015 (Pisa, Italy, May 11-14, 2015), pp. 1723-1728

[14] Collège Français de Métrologie, 27 exemples d'évaluation d'incertitudes d'étalonnage, Les guides techniques du Collège Français de Métrologie (Lexitis, Paris, France, 2014), ISBN 978-2-36233-127-5 9782362331275

[15] A. Ferrero, S. Salicone, IEEE Trans. Instrum. Meas. 58, 365 (2009)

[16] A. Ferrero, M. Prioli, S. Salicone, IEEE Trans. Instrum. Meas. 64 (2015) 